\title{
Autophagy downregulation contributes to insulin resistance mediated injury in insulin receptor knockout podocytes in vitro
}

Ying Xu, Qi Zhou, Wei Xin, Zhaoping Li, Liyong Chen, Qiang Wan

It is unknown whether autophagy activity is altered in insulin resistant podocytes and whether autophagy could be a therapeutic target for diabetic nephropathy (DN). Here we used shRNA transfection to knockdown insulin receptor (IR) gene in cultured human immortalized podocytes as an in vitro insulin resistant model. Autophagy related proteins LC3, Beclin, and p62 as well as nephrin, a podocyte injury marker were assessed using western blot and immunofluorescence staining. Our results show that autophagy is suppressed when podocytes lose insulin sensitivity and that treatment of rapamycin, an mTOR specific inhibitor, could attenuate insulin resistance induced podocytes injury via autophagy activation. The present study deepens our understanding of the role of autophagy in the pathogenesis of DN. 
1 Autophagy downregulation contributes to insulin resistance mediated injury in insulin receptor

2 knockout podocytes in vitro

3

4 Ying $\mathrm{Xu}^{1 *}$, Qi Zhou ${ }^{2 *}$, Wei Xin ${ }^{3}$, Zhaoping $\mathrm{Li}^{4}$, Liyong Chen ${ }^{5}$, and Qiang Wan ${ }^{6 \#}$

6 From ${ }^{1}$ Department of Nephrology, Shandong Provincial Hospital Affiliated to Shandong

7 University, Jinan, China; ${ }^{2}$ School of Medicine, Shandong University, Jinan, China; ${ }^{3}$ Department

8 of Central Laboratory, Shandong Provincial Hospital Affiliated to Shandong University, Jinan,

9 China; ${ }^{4}$ School of Public Health, Shandong University; ${ }^{5}$ Department of Nutrition, Shandong

10 Provincial Hospital Affiliated to Shandong University, Jinan, China; ${ }^{6}$ Department of Nephrology,

11 Shandong Provincial Qianfoshan Hospital Affiliated to Shandong University, Jinan, Shandong,

12 China.

13 *These authors contribute equally.

14 \#To whom correspondence should be addressed. Qiang Wan, Department of Nephrology,

15 Shandong Provincial Qianfoshan Hospital Affiliated to Shandong University, Jinan, China, Tel:

16 (86) 15168888915; Email: wanyanshaoqiang@163.com;

17 Running head: Insulin resistance downregulates autophay 


\section{Abstract:}

20 It is unknown whether autophagy activity is altered in insulin resistant podocytes and whether

21 autophagy could be a therapeutic target for diabetic nephropathy (DN). Here we used shRNA

22 transfection to knockdown insulin receptor (IR) gene in cultured human immortalized podocytes

23 as an in vitro insulin resistant model. Autophagy related proteins LC3, Beclin, and p62 as well as

24 nephrin, a podocyte injury marker were assessed using western blot and immunofluorescence

25 staining. Our results show that autophagy is suppressed when podocytes lose insulin sensitivity

26 and that treatment of rapamycin, an mTOR specific inhibitor, could attenuate insulin resistance

27 induced podocytes injury via autophagy activation. The present study deepens our understanding

28 of the role of autophagy in the pathogenesis of DN. 
30

31

32

\section{Introduction}

Diabetic nephropathy (DN) is the leading cause of end-stage kidney disease (ESRD) worldwide. It is also a major devastating complication of diabetes mellitus (DM), with up to $40 \%$ of diabetic patients experiencing this problem (Shi and $\mathrm{Hu}, 2014)$. With the rapidly increasing prevalence of DM being a major global health issue, it becomes more and more important to find therapeutic interventions directed at preventing the development and progression of DN.

$$
\text { The natural history of DN is dominated by progressive albuminuria, and podocytes are key }
$$
components of the ultrafiltration system in the glomeruli. Moreover, podocyte number and morphology have been proved to be predictors of DN progression. This makes the glomerular podocyte an attractive early target cell. Molecular mechanisms involved in the etiology and progression of DM and its complications have been studied intensively. Among them, insulin resistance was proved to be a critical one. In glomeruli of obese and diabetic rats, insulin sensitivity was reduced (Mima et al., 2011). Previous studies have shown that podocytes are insulin responsive cells in glomeruli (Madhusudhan et al., 2015; Tejada et al., 2008), and a loss of podocyte insulin sensitivity in perfused glomerulus results in an albuminuric phenotype even under normal glycemic conditions (Coward et al., 2005). Thus, insulin signaling in podocytes is essential for normal glomerular function. This has been proved by studies that show specific deletion of podocyte insulin receptor (IR) causes significant proteinuria and glomerulosclerosis in mice (Welsh et al., 2010). However, how podocyte inuslin resistance leads to podocytes injury remains unclear. 
50

51

52

Autophagy is an intracellular catabolic process by which aggregates and malfunctioned organelles are degraded to maintain intracellular homeostasis (Levine and Ranganathan, 2010).

Defects in autophagy have been closely associated with many human diseases, including cancer, neurodegenerative disorders. Accumulating evidence suggests that regulation of autophagy system may become a new therapeutic option for treatment of DN (Kume et al., 2014). In both genetic and dietary mouse models of obesity and insulin resistance, decreased autophagy in hepatic cells has been observed (Yang et al., 2010). Moreover, in obese animals, the activation of autophagy could be protective metabolic abnormalities (He et al., 2012). However, it is worthy of notice that modulation of autophagy in DM varies in different cell types. In pancreatic $\beta$-cells, autophagy is activated due to peripheral insulin resistance, whereas autophagy is inhibited in the hepatic cells of Type 2 diabetes (T2D) mice with insulin resistance (Rovira-Llopis et al., 2015). Whether the process of autophagy in insulin resistant podocytes is altered is still an open question. There are also uncertainties about whether autophagy participates in the insulin resistance mediated podocyte injury. The present study aims at evaluating the role of autophagy in diabetic nephropathy with focus on podocyte insulin resistance. We hypothesize that blunted autophagy activity in insulin resistant podocyte is one of the mechanisms that accounts for the podocytes injury in the pathogenesis of diabetic nephropathy. To test our hypothesis, IR knockout podocytes were used as an in vitro insulin resistant model.

\section{Materials and methods}

2.1. Cell culture 
70 Conditionally immortalized human podocytes were obtained from professor Fan Yi of Shan

71 Dong University. The cells were maintained in RPMI 1640 medium (HyClone, USA) containing

$7210 \%$ heat-inactivated fetal calf serum (Hyclone, USA) and $100 \mathrm{U} / \mathrm{ml}$ penicillin(Hyclone,USA) in

73 the presence of $5 \% \mathrm{CO}_{2}$ as described (Saleem et al., 2002). The cells were cultured in $33^{\circ} \mathrm{C}$ to

74 sustain podocyte proliferation, then podocytes were cultured at $37^{\circ} \mathrm{C}$ for $10-14$ days to induce

75 differentiation. All experiments were performed on passages 10-14 differentiated podocytes in

76 the present study. Insulin $(200 \mathrm{nM})$ was added $30 \mathrm{~min}$ before cells were harvested.

77

78 2.2. Knockdown of insulin receptor (IR) by shRNA transfection.

79 Selected shRNA lentivirus vector (pGMLV-SC1) against IR or negative control shRNA with

80 eGFP were designed and purchased from Genomeditech Company (Shanghai, China). The

81 transfection was performed as previously described (Xu et al., 2014). Figure 1 shows the

82 successful downregulation of IR by IR shRNA transfection. Under the experimental conditions,

83 we routinely obtain $80 \%$ downregulation of IR (Fig. 1). Thus, IR shRNA transfection was used

84 as the in vitro model to study the role of autophagy in insulin resistant podocytes.

87 Total RNA was isolated from podocyte and reverse transcribed to cDNA by PrimeScript ${ }^{\mathrm{TM}} \mathrm{RT}^{\mathrm{T}}$

88 Reagent Kit with gDNA Eraser (Takara). Real time PCR was performed using the light cycler 
89480 (Roche Diagnostics) with SYBR Premix Ex Taq ${ }^{\text {TM }}$ II (Takara). The knockdown efficiency

90 of the IR was confirmed by real time quantitative PCR (RT-qPCR) using sequence-specific

91 primers for IR (forward 5'- GGAGCTGTCCTAGGTGCTGTTTC -3' and reverse 5'-

92 CTTGTGTCAGTTCCCACAGCTTC -3'), which were designed by TaKaRa. The expression

93 levels of IR were normalized to $\beta$-actin expression level (forward 5'-

94 TGGCACCCAGCACAATGAA -3' and reverse 5' - CTAAGTCATAGTCCGCCTAGAAGCA $\left.953^{\prime}\right)$.

96

97 2.4. Western blot analysis.

98 The procedure of western blot analysis was carried out as described previously (Delfin et al.,

99 2011). The following primary antibodies were used: antibodies for SQSTM1/p62 (Cell Signaling,

100 5114), LC3B (Abcam, ab48394), Beclin-1 (Santa Cruz, SC-11427), IR(Santa Cruz, sc-711) and

101 nephrin(Abcam, ab58968) Primary antibody against $\beta$-actin and horseradish peroxidase-

102 conjugated secondary antibodies were from ZSGB-BIO.

103

1042.5 Immunofluorescence staining

105 The immunofluorescence staining was performed using protocol modified from previous

106 publication (Ning et al., 2011). Briefly, cells were fixed in 4\% paraformaldehyde for $10 \mathrm{~min}$ at

107 room temperature, then washed and permeabilized using PBS containing $0.3 \%$ Triton X-100, 
108 followed by blocking with $5 \%$ BSA in PBS. 1:100 dilution primary antibodies were added to the

109 cells. After incubating overnight at $4^{\circ} \mathrm{C}$, cells were washed. $10 \mu \mathrm{g} / \mathrm{ml}$ Alexa Fluor488 or Alexa

110 Fluor594 secondary antibodies were added and incubated for $30 \mathrm{~min}$. Images were acquired by

111 immunofluorescence microscopy (Nikon Ti-S, Tokyo, Japan).

112 2.6 Electron microscopy

113 After treatments, podocytes were trypsinized and collected into centrifuge tubes after washing by

114 PBS. Then, the cells were fixed by $3 \%$ glutaraldehyde at $4^{\circ} \mathrm{C}$, dehydrated by dimethylketone.

115 After embedment in Epon-812, the samples were cut into ultrathin sections (70 nm). Uranium

116 acetate and plumbum citrate were used to dye the ultrathin sections. The samples were observed

117 with JEM-100sX electron microscopy.

118

119 2.7. Statistics.

120 Data are presented as means \pm SD unless stated otherwise. Data were analyzed using repeated

121 measurement ANOVA followed by t test when appropriate with two tailed $\mathrm{p}$-values $<0.05$

122 considered statistically significant. GraphPad Prism software (version 5) was used for data

123 analysis.

124

125 3. Results 
126 3.1. Autophagy activity was down-regulated in IR-knockdown podocytes.

127 To investigate autophagy activity under insulin resistant conditions, we examined the changes of

128 autophagy related protein abundances in IR deficient podocytes(Figure 1). Figure 2 shows the

129 western blots of Beclin1, p62 and LC3II, indicating that autophagy was downregulated in IR

130 knockdown podocytes. During the autophagosome formation, LC3-I is processed into a lapidated

131 LC3-II form. The LC3-II/LC3-I ratio is considered as a marker of auophagosome formation.

132 Chloroquine (CQ) was used as the lysosomal activity blocker to evaluate the autophagic

133 flux.Compared with control group, a significant less LC3-II was detected in the IR-knockdown

134 cells (Fig.2A). Similar to the LC3 protein, western blots showed decreased level of Beclin1 (a

135 marker for autophagosome initiation complex) (Fig.2B). Compared to control cells, IR

136 knockdown podocytes showed significant increased expression of $\mathrm{p} 62$, indicating decreased

137 autophagy activity (Fig.2B). Figure 3 shows the immunofluorescence of Beclin1, p62 and LC3.

138 In consistent with the western blot results, the immunofluorescence staining of Beclin1 and p62

139 also indicates a decreased autophagy activity in IR-knockdown podocytes. Figure 4 shows the

140 electron microscopy images of control and IR knockdown podocytes. There were significant

141 less autophagosomes in IR knockdown podocytes compared to control cells.

143 3.2. IR deficiency induced podocyte injury in vitro. 
144 Nephrin has been proved to be critical for the action of insulin on podocytes (Coward et al., 2007)

145 and its expression has been used as the marker of podocyte integrity. Figure 5 shows the nephrin

146 expression levels in control and IR knockdown podocytes. Both western blot (Fig. 5A) and

147 immunofluorescence staining (Fig. 5B) of nephrin show that IR-knockdown podocytes had a

148 significant decrease of nephrin expression level, indicating podocyte injury induced by insulin

149 resistance.

1503.3 Rapamycin ameliorated insulin resistance induced podocyte injury via autophagy activation.

151 Rapamycin has been clinically used to inhibit rejection after organ transplantation as a FDA

152 approved immunosuppressive drug. It works by binding to mammalian target of rapamycin

153 (mTOR) and inhibiting the activity of mTOR protein kinase (Hartford and Ratain, 2007).

154 Rapamycin has been shown to induce autophagy in many cell types and species (Huber et al.,

155 2011). Figure 6 shows the p62, LC3II and nephrin level in IR-deficient podocytes with or

156 without the treatment of rapamycin. DMSO was used as the control group since rapamycin was

157 resolved in DMSO. Compared to the DMSO control group, IR-deficient podocytes treated with

158 rapamycin showed elevated autophagy activity indicated by increased LC3II expression.

159 Meanwhile, nephrin expression was restored by rapamycin treatment. Our results indicate that

160 podocyte injury under insulin resistant conditions was ameliorated by rapamycin treatment via

161 activation of autophagy. 


\section{4. Discussion}

164 The main findings of the current study are that under insulin resistant conditions, autophagy

165 activity in podocytes was suppressed in vitro; Treatment of rapamycin, an mTOR specific

166 inhibitor, could attenuate insulin resistance induced podocytes injury via autophagy activation.

167 Our results show that autophagy related proteins such as Beclin1 and LC3 were down

168 regulated and p62 was increased in IR-knockdown podocytes. Thus, our data indicates decreased

169 autophagy activity is induced in podocytes under insulin resistant conditions. It has been

170 demonstrated previously that autophagy plays a renoprotective role in the kidney. Podocyte-

171 specific autophagy deficient mice generated by Atg5 gene deletion have glomerular lesions

172 accompanied by podocytes apoptosis and albuminuria with aging (Mizushima et al., 2004).

173 Greatly increased susceptibility to glomerular disease was exhibited in mice lacking Atg5 gene in

174 podocytes. These findings underscore the importance of autophagy regulation as a critical

175 mechanism to maintain podocyte homeostasis. With the evidence of autophagy in kidney health

176 and disease accumulating, studies to investigate the role of autophagy in DN have attracted

177 intensive interests. Histone deacetylase 4 inhibition could ameliorates podocyte injury and

178 attenuates glomerulopathy in DN, and the maintenance of autophagy in podocytes was suggested

179 to be the mechanism underlying (Wang et al., 2014). In patients and rats with diabetes,

180 insufficient podocyte autophagy was observed histologically. Moreover, podocyte-specific

181 autophagy-deficient mice developed podocytes loss and massive proteinuria in a high-fat diet-

182 induced diabetes model that usually presented with minimal proteinuria (Tagawa et al., 2016). 
183 These all underline the role of autophagy in podocytes in DN pathogenesis. Our study

184 contributes to the understanding of how autophagy activity responses to insulin resistance with

185 further detailed mechanisms to be investigated.

186 Podocyte insulin resistance is associated with glomerular podocyte dysfunction. In this study,

187 downregulated autophagy activity was demonstrated to participate in podocyte injury. Since

188 insulin resistance is a prevalent metabolic feature in DM, it is difficult to answer the question

189 that which happens first, the onset of insulin resistance or alterations of autophagy. High-fructose

190 feeding mice showed disruption of autophagy in the liver, which appeared as an early event

191 preceding the onset of insulin resistance. Drugs able to restore the autophagic flux could indeed

192 prevent insulin resistance (Wang et al., 2015). Importantly, a recent study found that autophagy

193 regulates muscle glucose homeostasis and increase insulin sensitivity (Tam and Siu, 2014).

194 However, most of the works about autophagy and insulin resistance were done in adipose tissue,

195 skeletal muscles or liver, and what the relationship in the kidney remains unknown. Our results

196 show that after IR knockdown in podocyte, there are decreased autophagy activities. With the

197 current study, we report a decreased autophagy that was poised in an in vitro setting of insulin

198 resistance. Hence, a reciprocal interaction between podocyte insulin resistance and autophagy

199 activity should be proposed. Taken together, among mechanisms that might be altered in

200 podocytes under insulin resistant conditions, autophagy seems to play a role. Other mechanisms

201 such as oxidative stress and endoplasmic reticulum (ER) stress are also implicated. Recently, it

202 has been shown that ER stress (Madhusudhan et al., 2015) and mitochondrial function (Ising et 
203 al., 2015) are modulated by insulin signaling. In adipocytes in vitro, endoplasmic reticulum (ER)

204 stress causes insulin receptor (IR) down-regulation, which accounts for insulin resistance. While

205 inhibition of autophagy could alleviate the ER stress induced IR down-regulation (Zhou et al.,

206 2009). It is also proposed that in diabetes, autophagy system is activated in response to ER stress

207 induced insulin resistance(Zhang et al., 2015). These results indicate that autophagy regulation in

208 DN is a rather complicated process. The genes and signaling pathways that link autophagy and

209 insulin resistance as well as podocyte injury in vivo need to be delineated.

210 Our results showed that with manipulation, the activation of autophagy could protect the

211 podocyte from injuries induced by insulin resistance. Podocyte insulin resistance has been

212 considered to play a role in genesis and propagation of DN. The study of insulin signaling

213 pathways in podocyte has gained considerable momentum recently. Our results show that

214 treatment of rapamycin could attenuate insulin resistance induced podocyte injury via autophagy

215 activation. Rapamycin is an mTOR specific inhibitor, while mTOR is an evolutionarily

216 conserved serine/threonine kinase. The role of rapamycin in DN has been studied in a large

217 spectrum. Several recent studies have shown that mTORC1 signaling is highly activated in

218 podocytes of diabetic kidneys in human beings and animals. Studies by others show that

219 rapamycin ameliorated renal hypotrophy in mice model of diabetes (Sakaguchi et al., 2006;

220 Sataranatarajan et al., 2007). Our previous study also found that rapamycin attenuated high

221 glucose induced lipotoxicity and epithelial-to-mesenchymal transition (EMT)via autophagy

222 activation in proximal tubular cells (Xu et al., 2015). Taken together, the present study further 
223 provides evidence for use of rapamycin to treat DN.

224 In summary, we found under insulin resistant conditions, autophagy activity in podocytes is

225 downregulated in vitro, and activation of autophagy could prevent insulin resistance induced

226 podocyte injury. The present study deepens our understanding of the role of autophagy in the

227 pathogenesis of DN. However, since the development of DM and/or DN is a very complex

228 process, autophagy may have impact on each stage in the pathogenesis. Future research is

229 demanded to further clarify the roles of autophagy, which will provide more evidences for

230 autophagy to be a therapeutic target to prevent or alleviate development of DN. 
233 Coward, R.J., Welsh, G.I., Koziell, A., Hussain, S., Lennon, R., Ni, L., Tavare, J.M., Mathieson, 234 P.W., and Saleem, M.A. (2007). Nephrin is critical for the action of insulin on human 235 glomerular podocytes. Diabetes 56, 1127-1135.

236 Coward, R.J., Welsh, G.I., Yang, J., Tasman, C., Lennon, R., Koziell, A., Satchell, S., Holman, G.D., 237 Kerjaschki, D., Tavare, J.M., Matheieson, P.W., Saleem, M.A. (2005). The human glomerular 238 podocyte is a novel target for insulin action. Diabetes 54, 3095-3102.

239 Delfin, D.A., Xu, Y., Peterson, J.M., Guttridge, D.C., Rafael-Fortney, J.A., and Janssen, P.M. 240 (2011). Improvement of cardiac contractile function by peptide-based inhibition of NF241 kappaB in the utrophin/dystrophin-deficient murine model of muscular dystrophy. Journal 242 of translational medicine $9,68$.

243 Hartford, C.M., and Ratain, M.J. (2007). Rapamycin: something old, something new, 244 sometimes borrowed and now renewed. Clinical pharmacology and therapeutics 82, 381245388.

246 He, C., Bassik, M.C., Moresi, V., Sun, K., Wei, Y., Zou, Z., An, Z., Loh, J., Fisher, J., Sun, Q., 247 Korsmeyer, S., Packer, M. May, H.I, Hill, J.A., Virgin, H.W., Gilpin, C., Xiao, G., Bassel-Duby, R., 248 Scherer, P.E., Levine, B. (2012). Exercise-induced BCL2-regulated autophagy is required for 249 muscle glucose homeostasis. Nature 481, 511-515.

250 Huber, T.B., Walz, G., and Kuehn, E.W. (2011). mTOR and rapamycin in the kidney: signaling 251 and therapeutic implications beyond immunosuppression. Kidney international 79, 502252511.

253 Ising, C., Koehler, S., Brahler, S., Merkwirth, C., Hohne, M., Baris, O.R., Hagmann, H., Kann, M., 254 Fabretti, F., Dafinger, C., Bloch, W., Schermer, B., Linermann, A., Bruning, J.C., Kurschat, C.E., 255 Muller, R.U., Wiesner, R.J., Langer, T., Benzing, T., Brinkkoetter, P.T. (2015). Inhibition of 256 insulin/IGF-1 receptor signaling protects from mitochondria-mediated kidney failure. 257 EMBO Mol Med 7, 275-287.

258 Kume, S., Yamahara, K., Yasuda, M., Maegawa, H., and Koya, D. (2014). Autophagy: emerging 259 therapeutic target for diabetic nephropathy. Seminars in nephrology 34, 9-16.

260 Levine, B., and Ranganathan, R. (2010). Autophagy: Snapshot of the network. Nature 466, $26138-40$. 
262 Madhusudhan, T., Wang, H., Dong, W., Ghosh, S., Bock, F., Thangapandi, V.R., Ranjan, S.,

263 Wolter, J., Kohli, S., Shahzad, K., Heidel, F., Krueger, M., Schwenger, V., Moeller, M.J.,Kalinski,

264 T., Reiser, J., Chavakis, T., Isermann, B. (2015). Defective podocyte insulin signalling

265 through p85-XBP1 promotes ATF6-dependent maladaptive ER-stress response in diabetic

266 nephropathy. Nat Commun 6, 6496.

267 Mima, A., Ohshiro, Y., Kitada, M., Matsumoto, M., Geraldes, P., Li, C., Li, Q., White, G.S., Cahill,

268 C., Rask-Madsen, C. King, G.L. (2011). Glomerular-specific protein kinase C-beta-induced

269 insulin receptor substrate-1 dysfunction and insulin resistance in rat models of diabetes

270 and obesity. Kidney international 79, 883-896.

271 Mizushima, N., Yamamoto, A., Matsui, M., Yoshimori, T., and Ohsumi, Y. (2004). In vivo

272 analysis of autophagy in response to nutrient starvation using transgenic mice expressing a

273 fluorescent autophagosome marker. Molecular biology of the cell 15, 1101-1111.

274 Ning, H., Lin, G., Lue, T.F., and Lin, C.S. (2011). Mesenchymal stem cell marker Stro-1 is a 75

275 kd endothelial antigen. Biochemical and biophysical research communications 413, 353-

276357.

277 Rovira-Llopis, S., Diaz-Morales, N., Banuls, C., Blas-Garcia, A., Polo, M., Lopez-Domenech, S., 278 Jover, A., Rocha, M., Hernandez-Mijares, A., and Victor, V.M. (2015). Is Autophagy Altered in 279 the Leukocytes of Type 2 Diabetic Patients? Antioxid Redox Signal 23, 1050-1056.

280 Sakaguchi, M., Isono, M., Isshiki, K., Sugimoto, T., Koya, D., and Kashiwagi, A. (2006).

281 Inhibition of mTOR signaling with rapamycin attenuates renal hypertrophy in the early

282 diabetic mice. Biochemical and biophysical research communications 340, 296-301.

283 Saleem, M.A., O'Hare, M.J., Reiser, J., Coward, R.J., Inward, C.D., Farren, T., Xing, C.Y., Ni, L., 284 Mathieson, P.W., and Mundel, P. (2002). A conditionally immortalized human podocyte cell 285 line demonstrating nephrin and podocin expression. Journal of the American Society of 286 Nephrology : JASN 13, 630-638.

287 Sataranatarajan, K., Mariappan, M.M., Lee, M.J., Feliers, D., Choudhury, G.G., Barnes, J.L., and 288 Kasinath, B.S. (2007). Regulation of elongation phase of mRNA translation in diabetic 289 nephropathy: amelioration by rapamycin. The American journal of pathology 171,17332901742.

291 Shi, Y., and Hu, F.B. (2014). The global implications of diabetes and cancer. Lancet 383, 292 1947-1948. 
293 Tagawa, A., Yasuda, M., Kume, S., Yamahara, K., Nakazawa, J., Chin-Kanasaki, M., Araki, H., 294 Araki, S., Koya, D., Asanuma, K., Kim, E.H., Haneda, M., Kajiwaa, N., Hayashi, K., Ohashi, H., 295 Ugi, S., Maegawa, H., Uzu, T. (2016). Impaired Podocyte Autophagy Exacerbates Proteinuria 296 in Diabetic Nephropathy. Diabetes 65, 755-767.

297 Tam, B.T., and Siu, P.M. (2014). Autophagic cellular responses to physical exercise in 298 skeletal muscle. Sports Med 44, 625-640.

299 Tejada, T., Catanuto, P., Ijaz, A., Santos, J.V., Xia, X., Sanchez, P., Sanabria, N., Lenz, O., Elliot, 300 S.J., and Fornoni, A. (2008). Failure to phosphorylate AKT in podocytes from mice with 301 early diabetic nephropathy promotes cell death. Kidney international 73, 1385-1393.

302 Wang, H., Sun, R.Q., Zeng, X.Y., Zhou, X., Li, S., Jo, E., Molero, J.C., and Ye, J.M. (2015).

303 Restoration of autophagy alleviates hepatic ER stress and impaired insulin signalling 304 transduction in high fructose-fed male mice. Endocrinology 156, 169-181.

305 Wang, X., Liu, J., Zhen, J., Zhang, C., Wan, Q., Liu, G., Wei, X., Zhang, Y., Wang, Z., Han, H., Xu, H., 306 Bao, C., Song, Z., Zhang, X., Li, N., Yi, F. (2014). Histone deacetylase 4 selectively contributes 307 to podocyte injury in diabetic nephropathy. Kidney international 86, 712-725.

308 Welsh, G.I., Hale, L.J., Eremina, V., Jeansson, M., Maezawa, Y., Lennon, R., Pons, D.A., Owen, 309 R.J., Satchell, S.C., Miles, M.J., Caunt, C.J., McArdle, C.A., Pavenstadt, H., Tavare, J.M., 310 Herzenberg, A.M., Kahn, C.R., Mathieson, P.W., Quaggin, S.E., Saleem, M.A. Coward, R.J. 311 (2010). Insulin signaling to the glomerular podocyte is critical for normal kidney function. 312 Cell metabolism 12, 329-340.

313 Xu, Y., Huang, J., Xin, W., Chen, L., Zhao, X., Lv, Z., Liu, Y., and Wan, Q. (2014). Lipid 314 accumulation is ahead of epithelial-to-mesenchymal transition and therapeutic 315 intervention by acetyl-CoA carboxylase 2 silence in diabetic nephropathy. Metabolism: 316 clinical and experimental 63, 716-726.

317 Xu, Y., Liu, L., Xin, W., Zhao, X., Chen, L., Zhen, J., and Wan, Q. (2015). The renoprotective role 318 of autophagy activation in proximal tubular epithelial cells in diabetic nephropathy. Journal 319 of diabetes and its complications.

320 Yang, L., Li, P., Fu, S., Calay, E.S., and Hotamisligil, G.S. (2010). Defective hepatic autophagy 321 in obesity promotes ER stress and causes insulin resistance. Cell metabolism 11, 467-478.

322 Zhang, N., Cao, M.M., Liu, H., Xie, G.Y., and Li, Y.B. (2015). Autophagy regulates insulin 323 resistance following endoplasmic reticulum stress in diabetes. J Physiol Biochem 71, 319- 
324327.

325 Zhou, L., Zhang, J., Fang, Q., Liu, M., Liu, X., Jia, W., Dong, L.Q., and Liu, F. (2009). Autophagy326 mediated insulin receptor down-regulation contributes to endoplasmic reticulum stress327 induced insulin resistance. Mol Pharmacol 76, 596-603. 


\section{1}

Figure 1. Successful downregulation of IR by IR shRNA transfection.

(A)Western blots of IR after transfected with IR shRNA.(B)qRT-PCR analysis showed a 80\% knockdown efficiency of IR after transfected with IR shRNA .
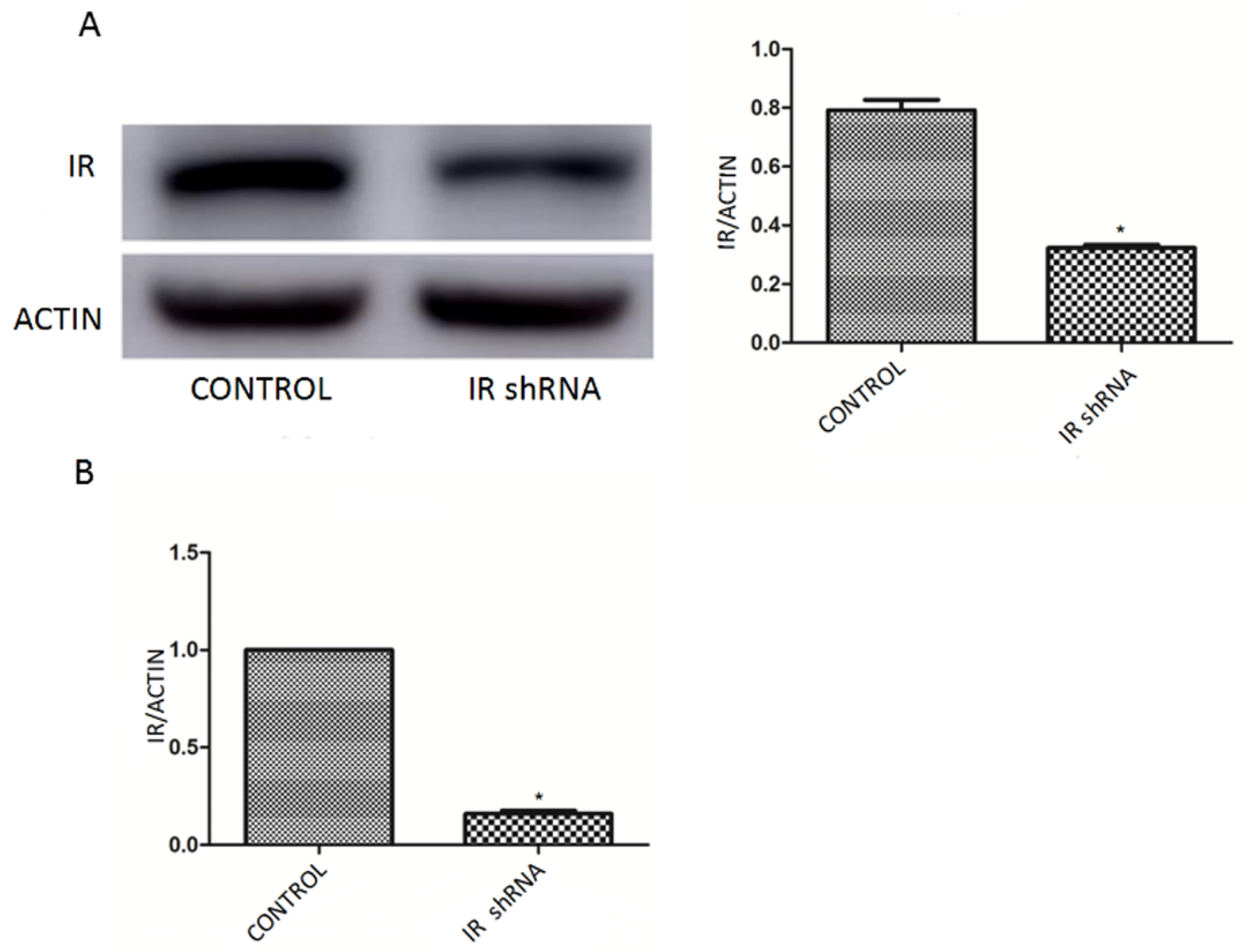
2

Figure 2.Autophagy was down-regulated after knockdown of IR.

(A)After 200nM insulin stimulation for 30min,the expression of LC3Ilwere decreased in cells transfected with IR shRNA with or without 50 uM chloroquine (CQ),compared with control shRNA.*P $<0.05$ vs. control shRNA.(B)After 200nM insulin stimulation for $30 \mathrm{~min}$, the expression of BECLIN1 were decreased, but P62 was up-regulated in cells transfected with IR shRNA, compared with control shRNA. $* P<0.05$ vs. control shRNA.

A
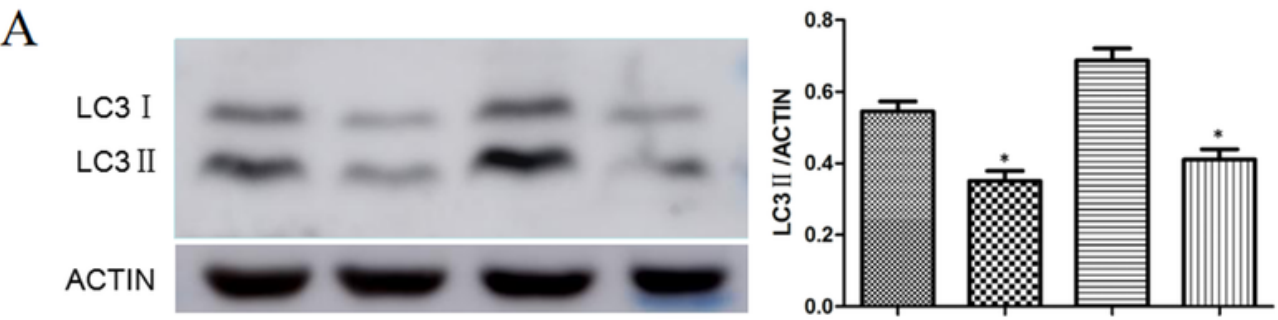

$\mathrm{B}$
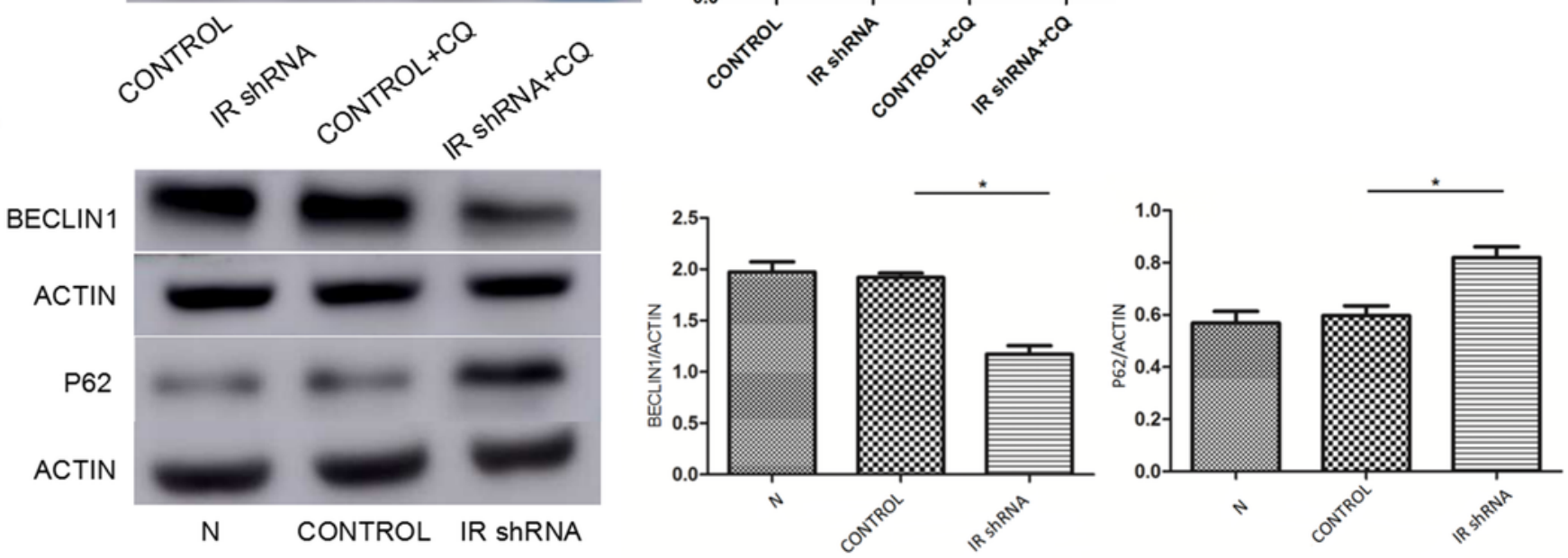


\section{3}

Figure 3.Immunofluorescence of autophagy markers shows that autophagy was downregulated after knockdown of IR.

After 200nM insulin stimulation for 30min, the staining of BECLIN1 and LC3IIwas decreased but P62 was enhanced in cells transfected with IR shRNA , compared with cells transfected with control shRNA .

BECLIN 1

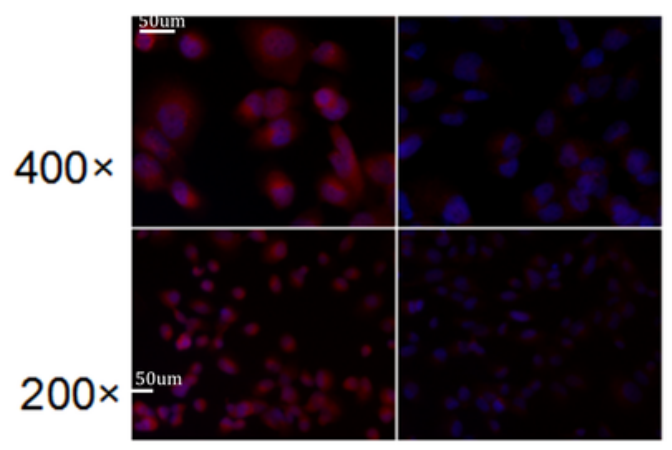

CONTROL IR ShRNA
P62

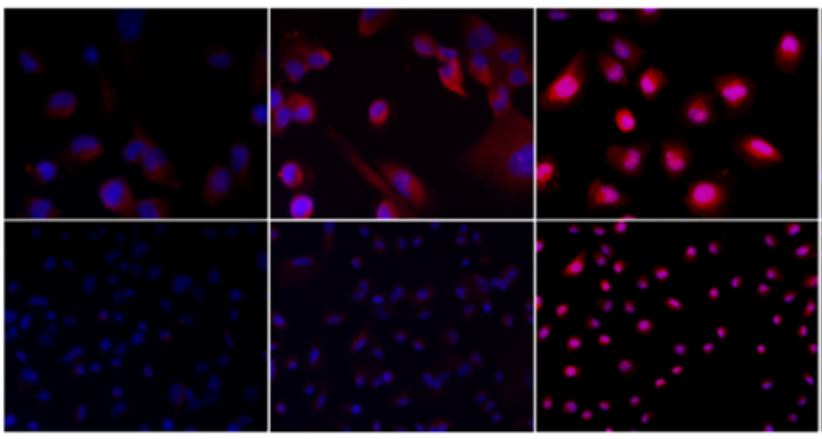

CONTROL IR shRNA
LC3

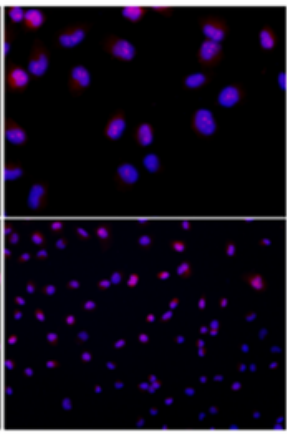

IR shRNA 
4

Figure 4.Electronic microscopy analysis of podocyte shows that autophagy was downregulated after knockdown of IR.

After $200 \mathrm{nM}$ insulin stimulation for $30 \mathrm{~min}$, the number of autophagosomes in cells transfected with IR shRNA was decreased compared with cells transfected with control shRNA .Black arrows indicate autophagosomes. Magnification $\times 15000$.

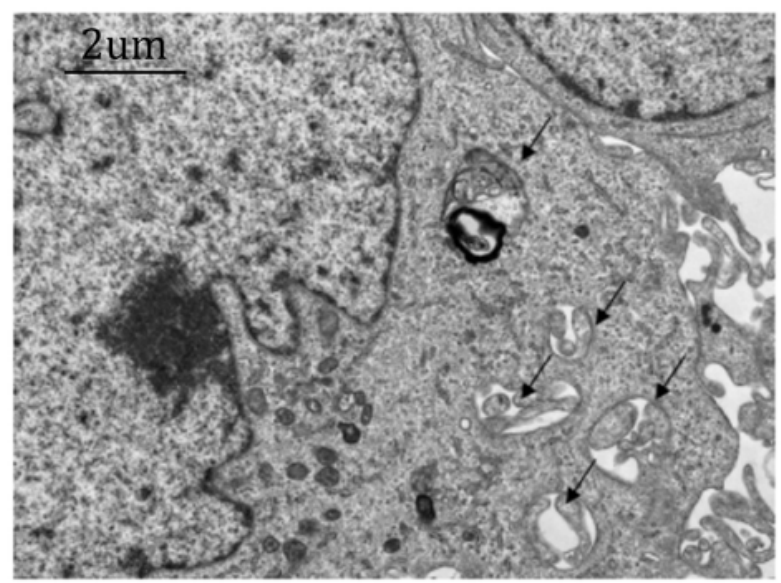

CONTROL

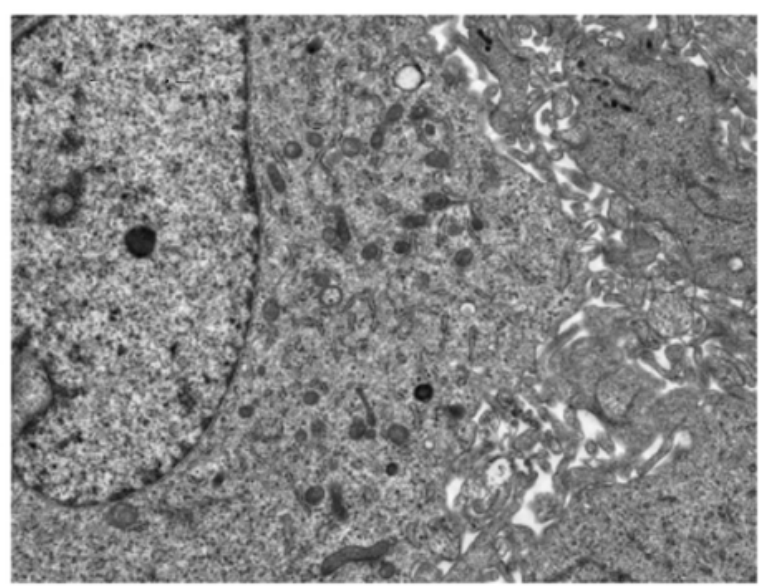

IR ShRNA 


\section{5}

Figure 5.Nephrin expression was down-regulated after knockdown of IR.

(A)WB showed after 200nM insulin stimulation for $30 \mathrm{~min}$, the expression of NEPHRIN was down-regulated in cells transfected with IR shRNA, compared with cells transfected with control shRNA *P $<0.05$ vs. control shRNA. (B) Immunofluorescence demonstrated that after 200nM insulin stimulation for 30min, NEPHRIN staining was decreased in cells transfected with IR shRNA (right), compared with cells transfected with control shRNA (left).

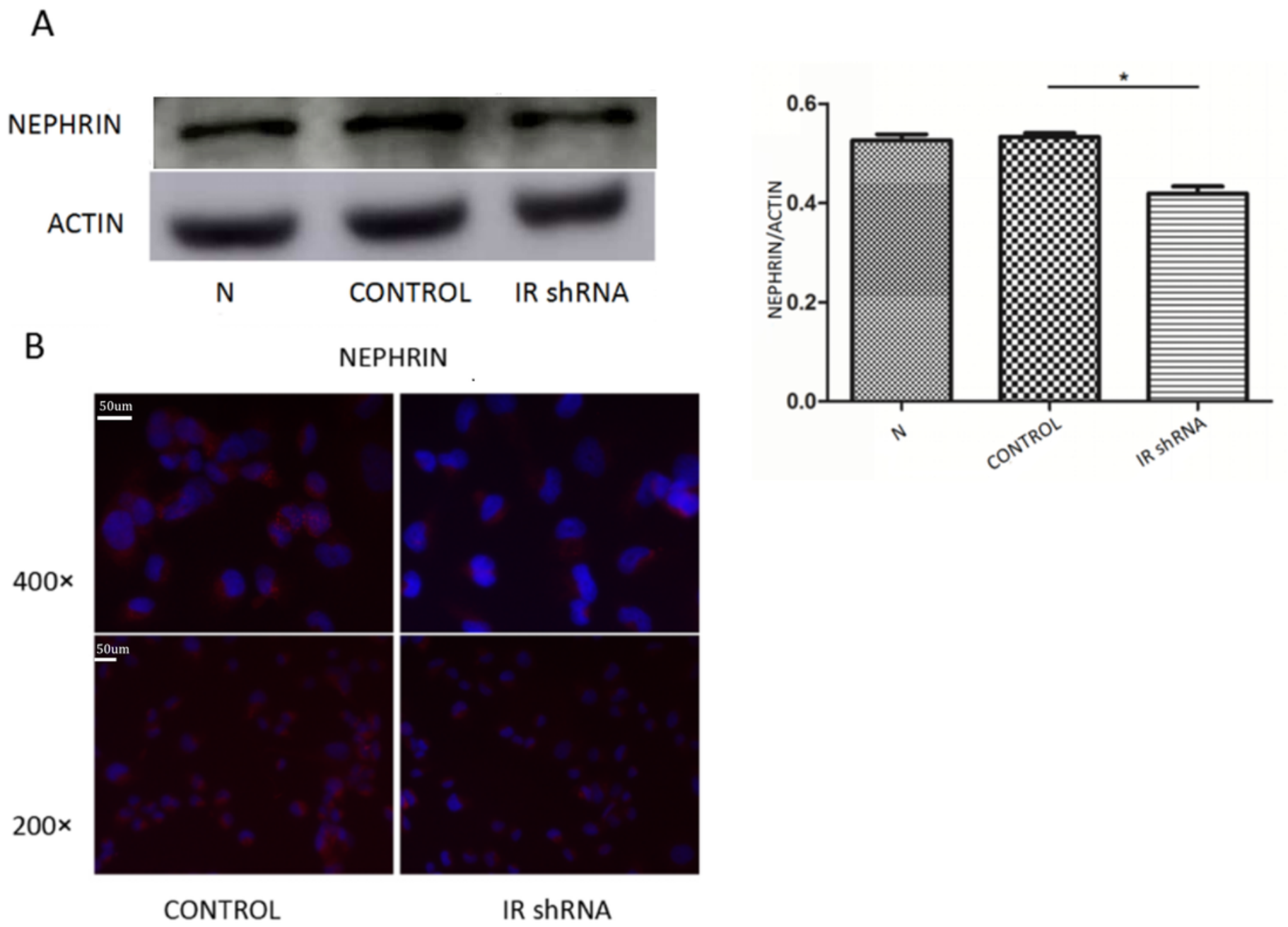


6

Figure 6.Rapamycin (RAPA) activated autophagy in IR-knockdown podocytes and increased the expression of Nephrin.

(A) After 10uM Rapamycin (RAPA) stimulation for $2 \mathrm{~h}$, the expression of p62 in IR-knockdown podocytes were decreased while NEPHRIN was up-regulated compared with cells without RAPA. *P $<0.05$ vs. IR shRNA.(B)After 10uM Rapamycin (RAPA) stimulation for $2 \mathrm{~h}$ the expression of LC3llin IR-knockdown podocytes was up-regulated in cells with or without CQ compared with cells without RAPA.. $* P<0.05$ vs. IR shRNA.

A

B
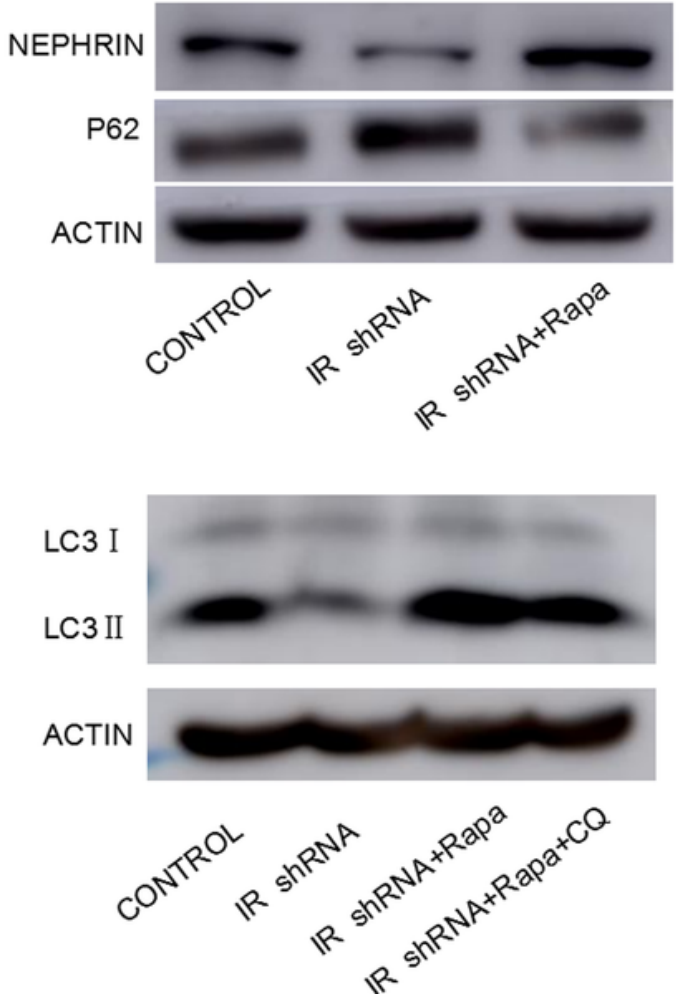
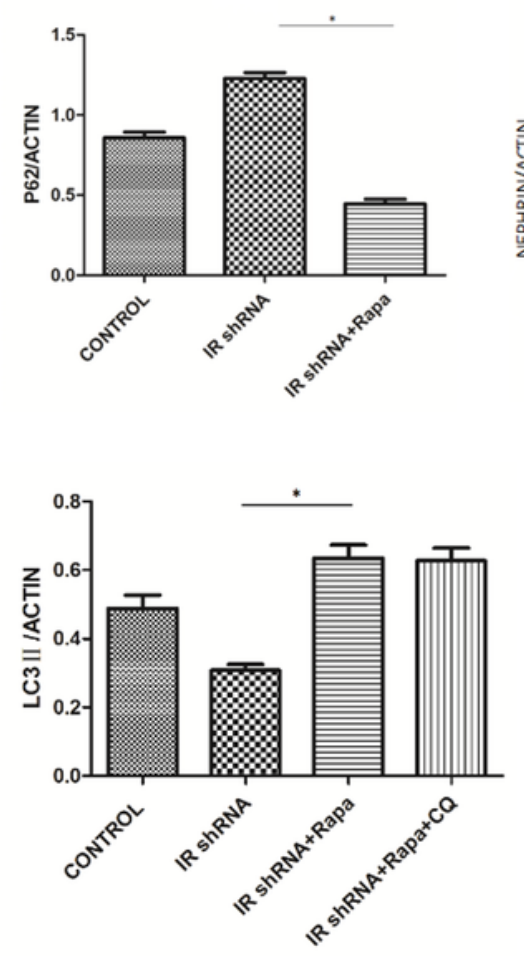

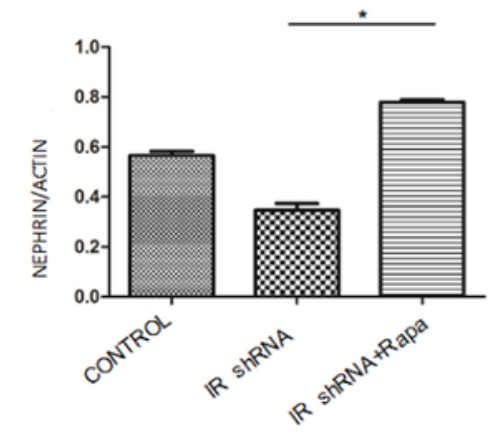

